\title{
Design, Manufacturing, and Inspection Data for a Three-Component Assembly
}

\author{
Thomas D. Hedberg, Jr. ${ }^{1}$, Michael E. Sharp ${ }^{1}$, Toby M. M. Maw ${ }^{2}$, Mostafizur M. Rahman ${ }^{2}$, Swati \\ Jadhav $^{2}$, James J. Whicker ${ }^{2}$, Allison Barnard Feeney ${ }^{1}$, and Moneer Helu ${ }^{1}$ \\ ${ }^{1}$ National Institute of Standards and Technology, \\ Gaithersburg, MD 20899, USA \\ ${ }^{2}$ The Manufacturing Technology Centre (MTC) Limited, \\ Coventry, UK \\ tdh1@nist.gov
}

Data DOI: https://doi.org/10.18434/T4/1503154

Key words: computer-aided design (CAD); manufacturing data; MTConnect; product assembly; Quality Information Framework (QIF).

Accepted: February 7, 2019

Published: February 12, 2019

https://doi.org/10.6028/jres.124.004

\section{Summary}

To better understand and address the challenges faced in linking all stages of a manufacturing and design process, an investigative fabrication process was designed and enacted as part of a collaboration between the National Institute of Standards and Technology (NIST) ${ }^{1}$ and the Manufacturing Technology Centre (MTC) ${ }^{2}$. This collaboration sought to test selected open standards' ability to integrate the product-lifecycle stages of engineering design, manufacturing, and quality assurance through a thorough implementation of a small scale model-based enterprise (MBE). A dataset was generated as a result of the collaboration. This article provides details about the dataset and instructions for how to access and/or retrieve it.

\footnotetext{
${ }^{1}$ https://www.nist.gov

${ }^{2}$ http://www.the-mtc.org
} 


\section{Data Specifications}

\begin{tabular}{|l|l|}
\hline NIST Operating Unit & Engineering Laboratory, Systems Integration Division, Systems Engineering Group \\
\hline Format & See Section 3 \\
\hline Instrument & Computer numerically controlled machine tool and coordinate measurement system \\
\hline Spatial or Temporal Elements & N/A \\
\hline Data Dictionary & MTConnect @ http://www.mtconnect.org/documents QIF @ https://qifstandards.org/download \\
\hline Accessibility & Publicly available at: https://smstestbed.nist.gov/tdp/mtc \\
\hline License & For data included in the data set see: https://www.nist.gov/director/licensing \\
\hline
\end{tabular}

\section{Dataset Directory Structure}

The following list describes the directory structure and the data included in the dataset.

- Computer-aided Design (CAD)

- Digital product definition in SolidWorks 2016 ${ }^{4}$, in accordance with ASME Y14.41-2012 [1]

- Clean

- Raw output from machine tool adapters with white-space and recording errors cleaned

- Pipe-delimited in accordance with the MTConnect standard v1.3 [2]

\section{- FAIR}

- First article inspection reporting (FAIR) data in Quality Information Framework (QIF) 2.1 format [3]

- Data conforms to QIF Extensible Markup Language (XML) Schema Definition (XSD) (see http://qifstandards.org/download)

- Each file contains data for 20 instances of the same part

- The FAIR was completed at NIST Headquarters (Gaithersburg, Maryland, USA) and Mitutoyo North America Headquarters (Aurora, Illinois, USA)

\section{- MTConnect}

- XML-formatted data of the machine tool output data in accordance with the MTConnect standard v1.3 [2]

- Data conforms to MTConnect v1.3 schemas (see https://github.com/mtconnect/schema)

- NC-Code

- Machining programs for operations steps for each part in accordance with ISO 6983 [4]

- PartData

- Combined "Split" data organized into data blocks by part instance

- QIF-IncomingInspection

\footnotetext{
${ }^{3}$ For data-dictionary licensing and usage information, please refer to the MTConnect and QIF standards.

${ }^{4}$ Certain commercial software products are identified in this paper. Such identification does not imply recommendation or endorsement by NIST, nor does it imply that the products identified are necessarily the best available for the purpose.
} 
- Receiving / incoming inspection reporting data in QIF 2.1 format [3]

- Data conforms to QIF XSD (see http://qifstandards.org/download)

- Each file contains data for 20 instances of the same part

- The receiving / incoming inspection was completed at The MTC (Coventry, United Kingdom)

- Raw

- Raw output from machine tool adapters as recorded on the given date and machine

- Split

- Parsed "Clean" data split into data blocks by operation for each part instance

\section{Impact}

This dataset adds to the Smart Manufacturing Systems (SMS) Test Bed's repository of technical data packages currently available to the public. The goal of the SMS Test Bed is to extend existing production-focused concepts by designing and architecting a test bed that enables smart manufacturing research and development across the product lifecycle. This process should highlight the challenges and requirements for introducing cyber-physical infrastructure in manufacturing, as well as create opportunities to provide a tangible source of data that other researchers may use to develop and validate smart manufacturing technologies. It also requires the integration of systems from each product lifecycle stage so that information may flow between design, fabrication, and inspection. This enables the development of technologies and standards that create a "digital thread" to exchange information across the product lifecycle. These efforts complement existing research and development aimed at reducing cycle time, ensuring first-pass success, and improving the overall performance of the product design and manufacturing process.

\section{Acknowledgments}

We thank Capvidia, Origin International, and Mitutoyo for their generous support and use of their software for completing the inspection tasks of this project. We also wish to thank William Bernstein (NIST), Brian Weiss (NIST), and Vijay Srinivasan (NIST) for their review and comments.

\section{References}

[1] American Society of Mechanical Engineers (2012) Y14.41 - 2012 - Digital Product Definition Data Practices (ASME, New York, NY).

[2] MTConnect Institute (2014) MTConnect Standard Part 1 - Overview and Protocol (The Association For Manufacturing Technology, Arlington VA). Available at https://static1.squarespace.com/static/54011775e4b0bc1fe0fb8494/t/ 557f2897e4b04b2acdba80b5/1434396823825/mtc_part_1_overview_v1.3.pdf.

[3] Dimensional Metrology Standards Consortium (2014) Part 1: Overview and Fundamental Principles - Quality Information Framework (QIF) - An Integrated Model for Manufacturing Quality Information (American National Standards Institute, Washington, DC). Available at http://qifstandards.org/.

[4] International Organization for Standardization (2009) ISO 6983-1:2009 - Automation systems and integration - Numerical control of machines - Program format and definitions of address words - Part 1: Data format for positioning, line motion and contouring control systems (ISO, Geneva, Switzerland). Available at https://www.iso.org/standard/34608.html.

About the authors: The authors are researchers at the Manufacturing Technology Centre (MTC) and the National Institute of Standards and Technology (NIST). The MTC researchers focus on better use of data and 
analytics applied to metrology processes to support the whole product lifecycle. The NIST researchers focus on the integration of models, systems, and processes across the product lifecycle to enable a "digital thread" of information flow between each phase of the lifecycle. MTC was established in 2010 as an independent Research \& Technology Organization (RTO), based in the United Kingdom, with the objective of bridging the gap between academia and industry. NIST is an agency of the United States Department of Commerce. 